\title{
Delayed hemichorea syndrome associated with nonketotic hyperglycemia
}

\section{Síndrome de hemicoreia tardia associada ao estado hiperglicêmico não cetótico}

Tiago Aguiar, Renata Nogueira, Rafael Vidon, Marcos Martins da Silva, Péricles Maranhão-Filho

A 69-year-old woman was admitted for investigation of an acute-onset right hemichorea ${ }^{1}$. T1-weighted brain magnetic resonance imaging (MRI) showed hyperintensity in the left basal ganglia (Figs 1A and B). Although diabetic, she had no hyperglycemia. Interestingly, two weeks earlier, the patient was admitted due to nonketotic hyperglycemia. Brain computed tomography, previously reported as normal (Fig 1C), showed subtle hyperdensity in the left basal ganglia. However, there were no movement disorders. Choreic movements have been reported in patients with nonketotic hyperglycemia. Usually, dyskinesia and metabolic disturbance are concomitant. Rarely, as in this case, although pathophysiologically controversial, a delayed dyskinesia is found, and this possibility should be considered when evaluating acute chorea in diabetic patients ${ }^{2}$.
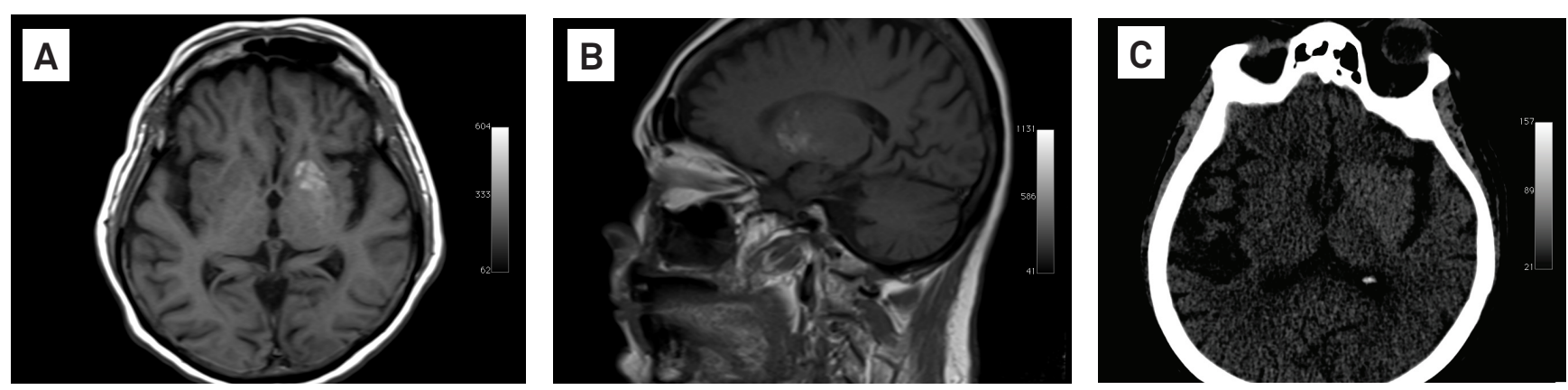

Fig 1. T1-weighted brain MRI showed classic image of hyperglycemia-induced lesion; there was no hyperglycemia but there were choreic movements (A, axial; B, sagittal). Two weeks earlier, when diagnosed with nonketotic hyperglycemia, brain computed tomography (C), prior reported as normal, showed subtle hyperdensity in the left basal ganglia, but no dyskinesia.

References

1. Postuma RB, Lang AE. Hemiballism: revisiting a classic disorder. Lancet Neurol 2003;2:661-668.
2. Ahlskog JE, Nishino H, Evidente VG, et al. Persistent chorea triggered by hyperglycemic crisis in diabetics. Mov Disord 2001;16:890-898.

Department of Neurology Clementino Fraga Filho Hospital from Federal University of Rio de Janeiro, Brazil.

Correspondence: Tiago Aguiar; Departamento de Neurologia, Hospital Universitário Clementino Fraga Filho/UFRJ; Avenida Brigadeiro Trompowiski s/n; 21941-590; Rio de Janeiro RJ - Brasil; E-mail: tiagosaguiar@gmail.com 